\title{
EXPERIMENTAL AND NUMERICAL INVESTIGATION OF THE SEISMIC BEHAVIOR OF RECTANGULAR TUNNELS IN SOFT SOILS
}

\author{
Grigorios Tsinidis $^{1}$, Kyriazis Pitilakis ${ }^{1}$, Charles Heron ${ }^{2}$ and Gopal Madabhushi ${ }^{2}$ \\ ${ }^{1}$ Aristotle University of Thessaloniki \\ Laboratory of Soil Mechanics, Foundations and Geotechnical Earthquake Engineering, POB 424, \\ 54124, Thessaloniki, Greece \\ e-mail: gtsinidi@civil.auth.gr,kpitilak@civil.auth.gr \\ ${ }^{2}$ University of Cambridge, Schofield Centre \\ High Cross, Madingley Road, Cambs CB3 0EL, Cambridge, UK \\ cmh78@cam.ac.uk, mspg1@cam.ac.uk
}

Keywords: Underground Structures, Rectangular Tunnels, Dynamic Centrifuge Tests, Dynamic Analysis

\begin{abstract}
Underground structures constitute crucial components of the transportation networks. Considering their significance for modern societies, their proper seismic design is of great importance. However, this design may become very tricky, accounting of the lack of knowledge regarding their seismic behavior. Several issues that are significantly affecting this behavior (i.e. earth pressures on the structure, seismic shear stresses around the structure, complex deformation modes for rectangular structures during shaking etc.) are still open. The problem is wider for the non-circular (i.e. rectangular) structures, were the soilstructure interaction effects are expected to be maximized. The paper presents representative experimental results from a test case of a series of dynamic centrifuge tests that were performed on rectangular tunnels embedded in dry sand. The tests were carried out at the centrifuge facility of the University of Cambridge, within the Transnational Task of the SERIES EU research program. The presented test case is also numerically simulated and studied. Preliminary full dynamic time history analyses of the coupled soil-tunnel system are performed, using ABAQUS. Soil non-linearity and soil-structure interaction are modeled, following relevant specifications for underground structures and tunnels. Numerical predictions are compared to experimental results and discussed. Based on this comprehensive experimental and numerical study, the seismic behavior of rectangular embedded structures is better understood and modeled, consisting an important step in the development of appropriate specifications for the seismic design of rectangular shallow tunnels.
\end{abstract}




\section{INTRODUCTION}

During past earthquakes, underground structures behaved generally better than near surface or aboveground structure. However, several cases of extensive damage and even collapse are reported in the literature [1], [2]. The most interesting case is that of the Daikai station in Kobe, Japan, that collapsed during the major Hyogoken-Nambu earthquake (1995) [3],[4]. This is actually, the first well-reported case of a total collapse of a large underground structure under seismic shaking. These recent failures revealed some important weaknesses in the current seismic design practices.

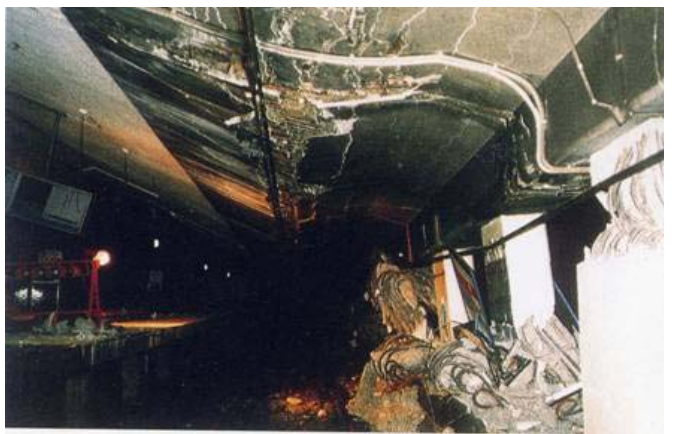

(a)

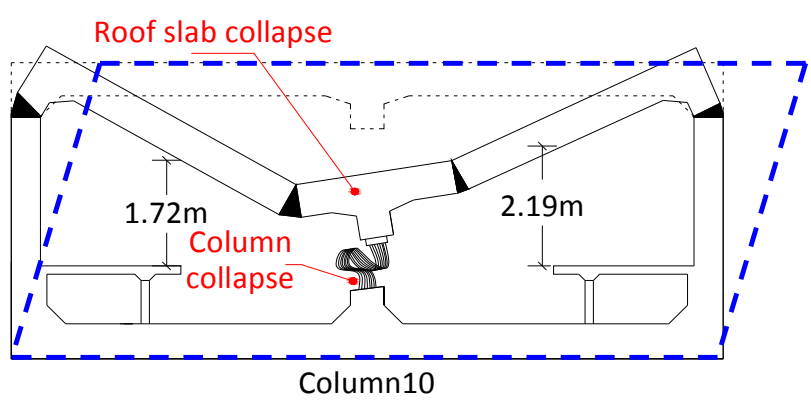

(b)

Figure 1: Daikai Station. (a) Collapse of the central columns of the station, [5], (c) Failure modes (modified after [3])

The seismic response of embedded structures to ground shaking is very distinct with respect to the aboveground structures. The kinematic loading imposed on the structure from the surrounding soil is prevailing, while the inertial effects are of secondary importance. The soilstructure interaction effects, that are expected to be increased in cases of non-circular (i.e. rectangular) embedded structures, are closely related to two crucial parameters, namely:

(i) the relative flexibility of the structure and the ground and

(ii) the interface characteristics between the structure and the surrounding soil.

The exact affection of these parameters on structural seismic response is not well known.

For the evaluation of the seismic response of underground structures, several methods may be found in the literature, ranging from uncoupled methods, simplified closed form solutions and equivalent static analysis schemes, to the most sophisticated full dynamic analysis of the coupled soil-structure system incorporating advanced numerical methods (i.e. [6-9] etc). The results of these methods may substantially deviate, even under the same assumptions, indicating the lack of knowledge regarding some very crucial issues that are significantly affecting the seismic response [10]. The lack of knowledge is even more pronounced for the noncircular structures. The seismic earth pressures on the side walls, the seismic shear stresses around the perimeter of the structure along with the soil-structure interface characteristics, the complex deformation modes during the shaking (i.e. rocking for stiff structures or inward deformations for the flexible structures) and the affection of the soil-structure relative flexibility on the seismic response are, among others, issues that need further study.

To this end, a comprehensive series of tests has been performed at the University of Cambridge (UCAM) jointly with Aristotle University of Thessaloniki (AUTH) on square tunnel models embedded in dry sand. The tests were carried out at the geotechnical centrifuge facility of the University of Cambridge, within the Transnational Access Task of the SERIES EU 
research program (TA project: TUNNELSEIS). The produced experimental data are used to better understand the seismic behavior of rectangular embedded structures and also to validate advance numerical models, improving at the end the design methods. The experimental procedure and the set up for one of the tests cases is briefly presented in the ensuing paper along with the some representative experimental results. Numerical predictions, achieved by preliminary numerical simulations of the test, are also briefly presented and compared to the experimental data.

\section{DYNAMIC CENTRIFUGE TESTS}

\subsection{Geotechnical facility}

The tests were carried out on a square aluminum tunnel-model embedded in dry sand, at the "Turner beam centrifuge" of the University of Cambridge (Schofield Centre), under centrifuge acceleration of $50 \mathrm{~g}$.

Earthquake input motions were applied using the Stored Angular Momentum (SAM) actuator [11], which is designed to apply sinusoidal input motions at a maximum frequency up to $60 \mathrm{~Hz}$ and at a maximum amplitude of $20 \mathrm{~g}$ (in model scale).

A large Equivalent Shear Box (ESB) was used as the container for the models, having inside dimensions $673 \mathrm{~mm}$ in length, $255 \mathrm{~mm}$ in width and $427 \mathrm{~mm}$ in depth. The box is designed to match the shear stiffness of the contained soil for the range of shear strains of interest, in order to minimize any soil-container interactions [12].
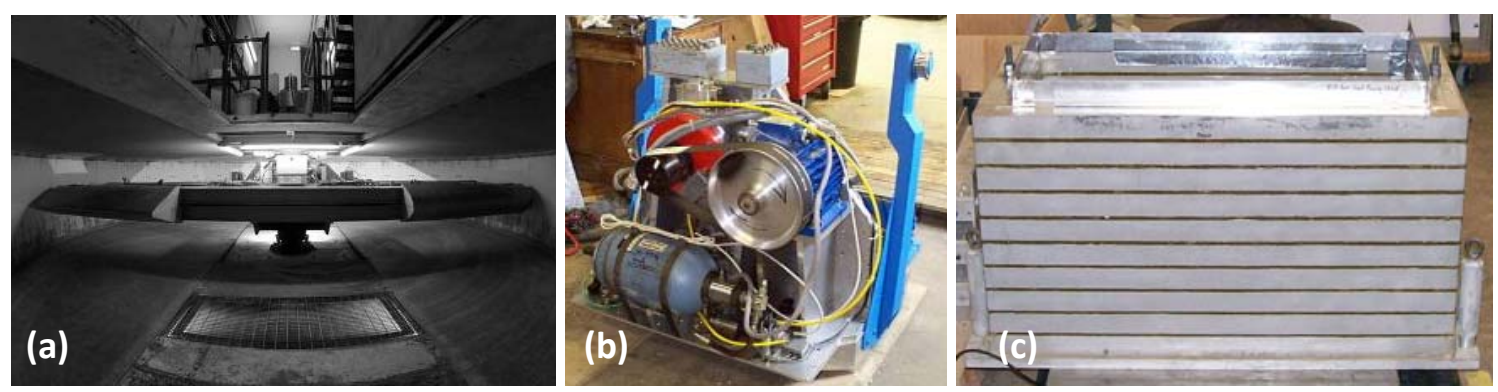

Figure 2: (a) Turner beam centrifuge, (b) SAM actuator, (c) ESB box

\subsection{Materials}

The model was made of dry uniform Hostun HN31 sand having a relative density of about $90 \%$. The mechanical properties of the sand are tabulated in Table 1.

The tunnel model (Figure 3), manufactured from $6063 \mathrm{~A}$ aluminum alloy, is $100 \mathrm{~mm}$ wide and $220 \mathrm{~mm}$ long, having a thickness of $2 \mathrm{~mm}$. The aluminum alloy mechanical properties are summarized in Table 2. According to the scale factor $(\mathrm{N}=50)$, the model corresponds to a $5 \times 5 \times 11(\mathrm{~m})$ square tunnel having an equivalent concrete lining thickness equal to $0.13 \mathrm{~m}$ (assuming $\mathrm{E}=30 \mathrm{GPa}$ for the concrete). This thickness is obviously unrealistic in practice, as the design analysis for the static loads will result in a much thicker lining. However, this selection was necessary to achieve measurements of the lining strains. To simulate more realistically the soil-structure interface, Hostun sand was stuck on the external face of the tunnel-model, creating a rough surface. 


\begin{tabular}{lllllll}
\hline & $\rho_{\mathrm{s}}\left(\mathrm{g} / \mathrm{cm}^{3}\right)$ & $\mathrm{e}_{\max }$ & $\mathrm{e}_{\min }$ & $\mathrm{d}_{10}(\mathrm{~mm})$ & $\mathrm{d}_{50}(\mathrm{~mm})$ & $\mathrm{d}_{60}(\mathrm{~mm})$ \\
\hline Hostun HN31 & 2.65 & 1.01 & 0.555 & 0.209 & 0.335 & 0.365 \\
\hline
\end{tabular}

Table 1: Sand mechanical properties (after [13])

\begin{tabular}{llll}
\hline $\begin{array}{l}\text { Unit weight, } \gamma \\
\left(\mathrm{kN} / \mathrm{m}^{3}\right)\end{array}$ & $\begin{array}{l}\text { Elastic modulus, E } \\
(\mathrm{GPa})\end{array}$ & $\begin{array}{l}\text { Poisson } \\
\text { ratio } \mathrm{v}\end{array}$ & $\begin{array}{l}\text { Tensile strength, } \\
\mathrm{f}_{\mathrm{bk}}(\mathrm{MPa})\end{array}$ \\
\hline 2.7 & 69.5 & 0.33 & 220 \\
\hline
\end{tabular}

Table 2: Tunnel model mechanical properties
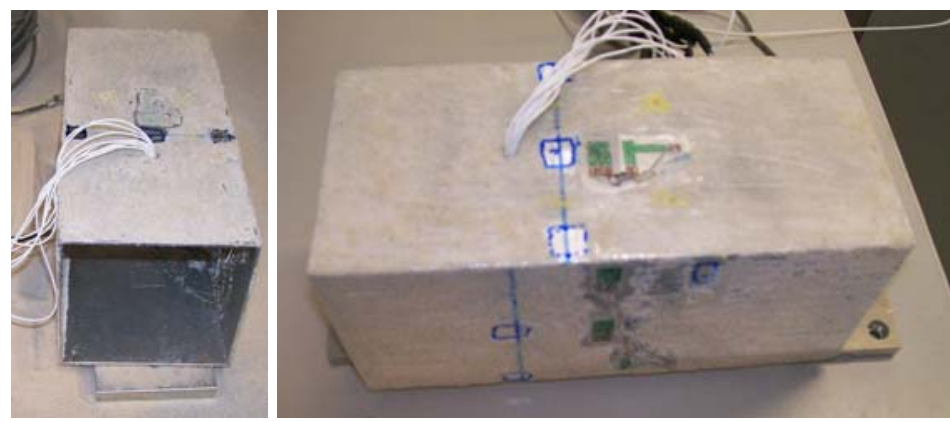

Figure 3: Tunnel model

\subsection{Model preparation}

The sand model was made using an automatic hopper system [14]. During the construction, the tunnel and all the embedded transducers were positioned in the model. Several phases of the model construction are presented in Figure 4. To avoid any interaction of the tunnel with the ESB box, the tunnel was shorter than the box width. Two PVC rectangular plates were placed at both the tunnel ends to avoid the sand entrance into the tunnel-model.
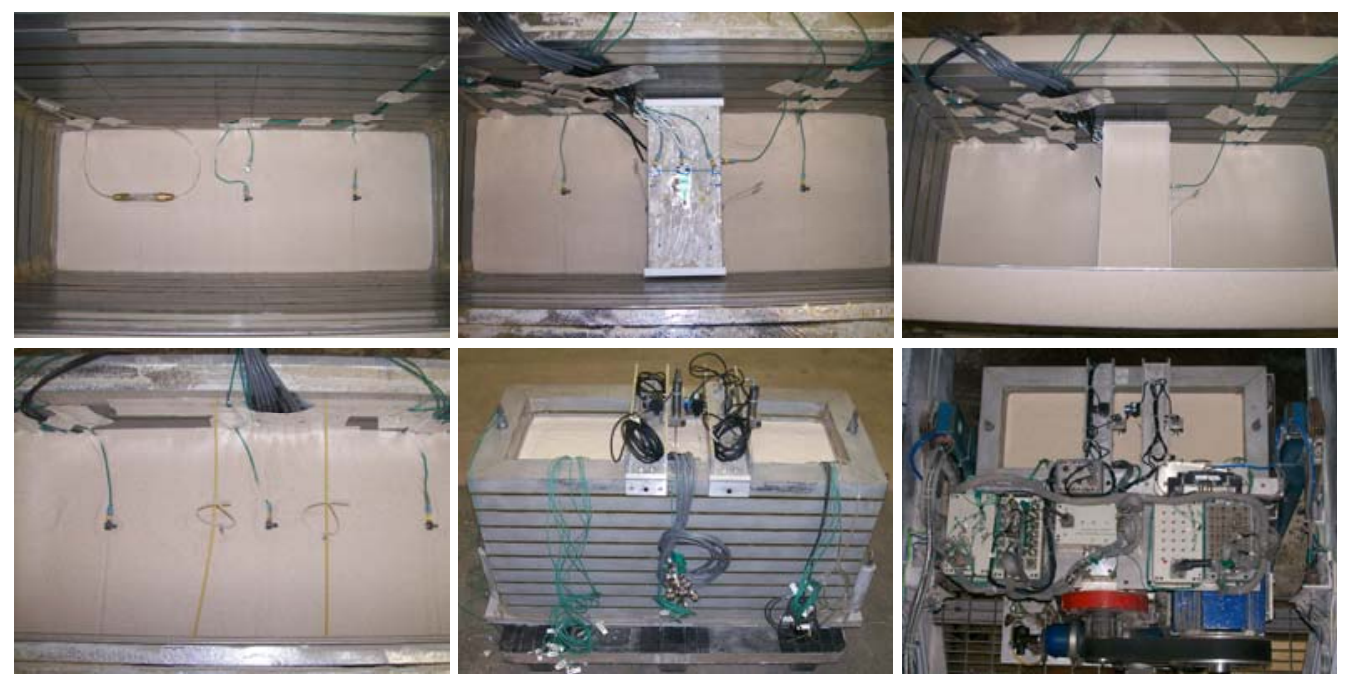

Figure 4: Model preparation 


\subsection{Model set up - instrumentation}

Figure 5 presents the final model layout and the instrumentation scheme. Miniature accelerometers were used to measure the acceleration in the soil, on the tunnel and on the ESB box. To estimate the soil shear wave velocity profile before each earthquake, air hammer tests were performed [15]. For this purpose, a small air-hammer was introduced close to the base of the soil layer while a set of accelerometers were placed above it, forming an array, allowing a record of the arrival times of the waves emanating from the air-hammer. The soil surface settlements were recorded in two locations using linear variable differential transformers (LVDTs), while two position sensors were attached on the upper side of the walls of the tunnel to capture the vertical displacement and the possible rocking of the tunnel model. Both the LVDTs and the POTs were attached on gantries running above the ESB box. Two miniature total earth pressure cells were attached on the left side wall of the tunnel, allowing the measurement of the soil earth pressures on the wall.

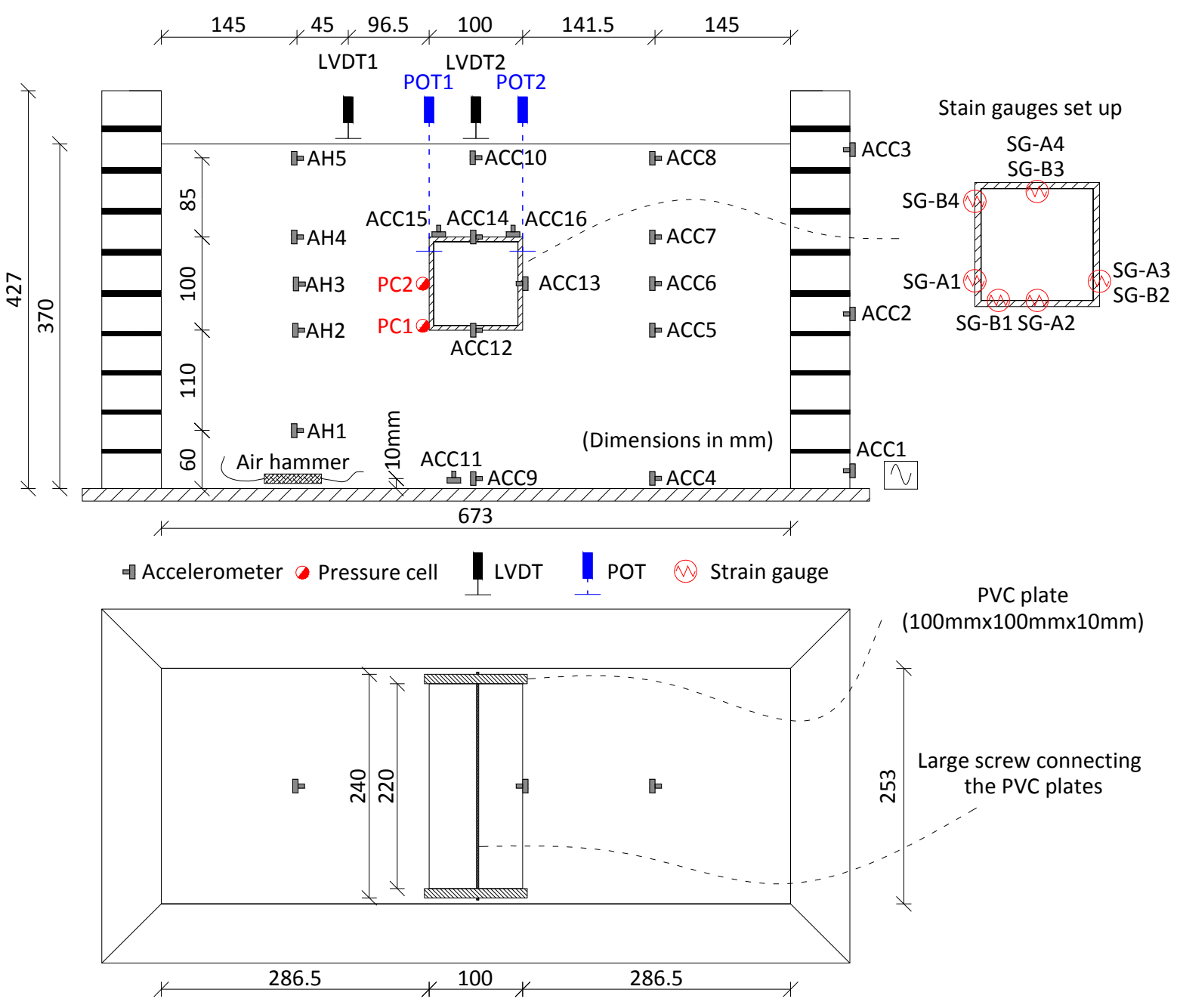

Figure 5: Model configuration - instrumentation scheme 
Resistance strain gauges were glued on the inner and the outer face of the tunnel to measure the model strains at several locations. Four of them were measuring the axial strains at the walls and the slabs, while four were recording the bending moment strains near the model corners and at the middle of the roof slab. The latter strain gauge was broken during the testing procedure. In the following presented results, positive values represent bending moment with tensile stress increments for the internal lining face and tensile axial force. The gauges cables were running from the inside face of the tunnel, to avoid any interactions with the soiltunnel interface. All the instruments were adequately calibrated before and checked after the tests, as described in detail in [16].

\subsection{Experimental procedure}

During each flight, the centrifuge was spun up in steps stopping at $10 \mathrm{~g}, 30 \mathrm{~g}$ and $50 \mathrm{~g}$ and then the earthquakes were fired in a row, leaving some time between them to acquire the data. The data was recorded at sampling frequency of $4 \mathrm{~Hz}$ during the swing up and at $4 \mathrm{kHz}$ during the earthquakes.

Before each shake, air-hammer tests were conducted to evaluate the new (modified) shear wave velocity $\left(\mathrm{V}_{\mathrm{s}}\right)$ profile of the soil deposit. The $\mathrm{V}_{\mathrm{s}}$ profiles were estimated based on the travel times of the waves, between accelerometers that are placed at known distances apart. These distances did not change significantly after each shake, as the recorded soil settlements were small due to the high relative density of the soil deposit. The travel times were estimated in a simplified way from the arrivals of the waves, produced by air-hammer. To make sure that the arrival times were adequately recorded, the DasyLab software was used as the acquisition system for the air-hammer array of accelerometers, allowing for a sampling frequency equal to $50 \mathrm{kHz}$.

The model was subjected to a total of eight "earthquakes" during two flights. The earthquakes were pseudo-harmonic wavelets except the last earthquake fired during the first flight that was a sine sweep. The main characteristics of the input motions are tabulated in Table 3 both in model and prototype scale (bracketed values), while the time histories are depicted in Figure 6 .
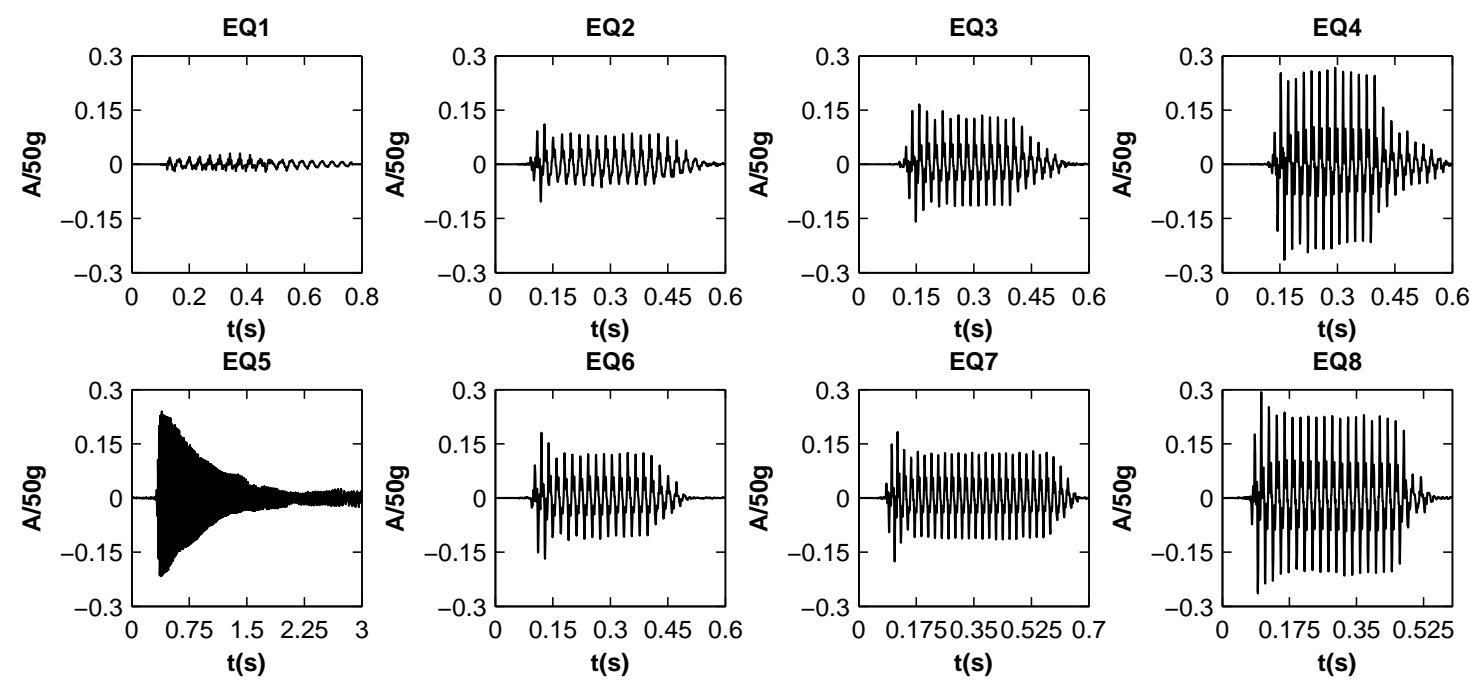

Figure 6: Input motions time histories 


\begin{tabular}{llll}
\hline \multicolumn{1}{c}{ Input type } & Frequency & Amplitude (g) & Nominal Duration (s) \\
\hline Flight 1 & & & \\
\hline EQ1 & $30\{0.6\}$ & $1.0\{0.02\}$ & \\
EQ2 Pseudo- & $45\{0.9\}$ & $4.0\{0.08\}$ & $0.4\{20\}$ \\
EQ3 Harmonic & $50\{1\}$ & $6.5\{0.13\}$ & \\
EQ4 & $50\{1\}$ & $12\{0.24\}$ & \\
EQ5 Sine Sweep & $60\{1.2\}$ & $12\{0.24\}$ & $3.0\{150\}$ \\
\hline Flight 2 & & & \\
\hline EQ6 & $50\{1\}$ & $5.8\{0.116\}$ & $0.4\{20\}$ \\
EQ7 Pseudo- & $50\{1\}$ & $6.0\{0.12\}$ & $0.6\{30\}$ \\
EQ8 Harmonic & $50\{1\}$ & $11.0\{0.22\}$ & $0.5\{25\}$ \\
\hline
\end{tabular}

Table 3: Input motions characteristics (bracketed values: values in prototype scale)

\subsection{Experimental results}

Representative experimental results are presented in the following sections comparatively to the numerical predictions, for EQ4. The main findings are summarized in the following:

- The horizontal acceleration recorded at several locations, is amplified towards the surface, while, the amplification ratio with respect to the base amplitude reduces with increased input motion amplitude due to the soil non-linear behavior.

- Vertical acceleration-time histories recorded on the sides of the model's roof slab were out of phase indicating a rocking mode of vibration for the tunnel.

- The tunnel's racking distortions, computed from the acceleration-time histories, indicate a rigid structure with respect to the surrounding soil.

- Small ground settlements were recorded at the soil surface during swing up and shaking, due to the high relative density of the studied soil deposit.

- During shaking the dynamic increments of the pressures were higher for the invert slabside wall corner due to the larger rigidity of the model at this location compared to the middle of the side-wall. Residual values are observed after each shake that can be mainly attributed to the soil plastic deformations and to a small amount of soil densification during shaking that can cause stress redistribution.

- The dynamic bending moments correspond in a similar way with the recorded dynamic earth pressures. Large residual values were detected after each shake, as a result of cumulative strains during the shaking.

- Smaller residuals were observed for the dynamic axial forces after shaking. These residuals in addition to the previously mentioned ones are most probably due to an amount of sliding observed on the soil-tunnel interface.

\section{NUMERICAL SIMULATION}

\subsection{Description of the numerical model}

For the numerical simulation of the test we used the generic FE code ABAQUS [18]. Full dynamic time history analyses of the coupled soil-tunnel system were performed, under plane strain conditions, on a prototype scale model. Appropriate scaling laws were used to convert the computed quantities from prototype to model scale [17]. 


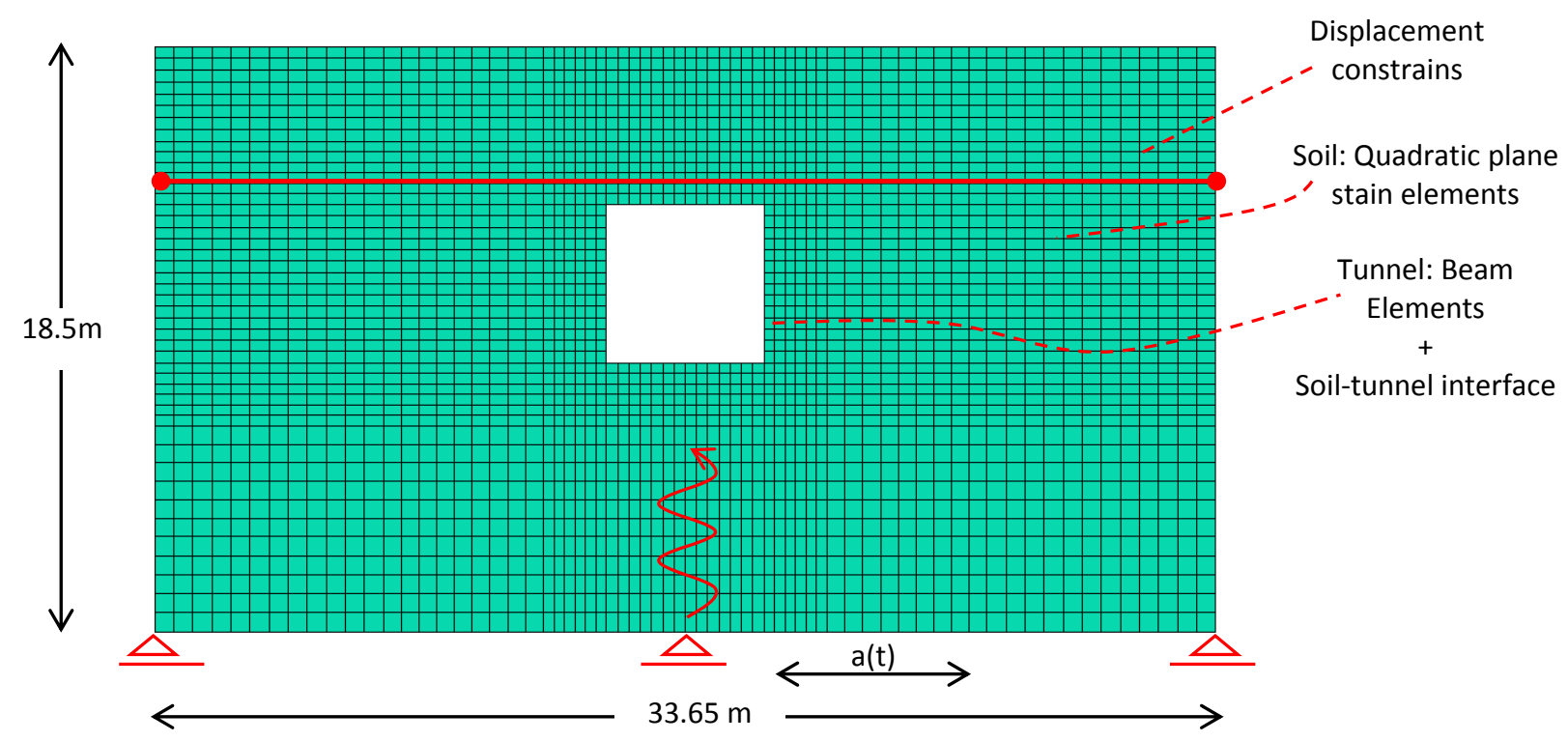

Figure 7: Numerical model in ABAQUS

More specifically, the soil was meshed with quadratic plane-strain elements, while the model tunnel was modeled with beam elements (Figure 7). Linear elastic behavior was assumed for the model tunnel $(\mathrm{E}=69.5 \mathrm{GPa}, \mathrm{v}=0.33)$, while the soil behavior was modelled with (i) a linear visco-elastic material (equivalent linear approach), (ii) a combined equivalent linear-elastoplastic approximation, using a Mohr-Coulomb failure criterion and (iii) a modified kinematic hardening model combined with a Von-Mises failure criterion and an associated plastic flow rule, as presented in detail in the following. The base boundary of the model was modelled as rigid bedrock, while kinematic constrains were introduced at the vertical boundaries, forcing the opposite vertical sides to move simultaneously preventing any rotation. The soil-tunnel interface was simulated using contact algorithms available in ABAQUS [18]. More specifically, the tangential behavior was modelled using the penalty friction formulation, introducing a friction coefficient $\mu=0.84$, considering the stack sand on the tunnel outer face. The normal surface behavior was adequately simulated, so to preclude penetration and allow separation between the two media, precluding tensile strains on the soil. The input motion was introduced at base of the model in terms of acceleration time histories, referring to the motion recorded at the reference accelerometer (see Figure 6). The analyses were performed in two steps; first the gravity loads were introduced, while in the second step the earthquake input motion was applied in a dynamic step.

Representative numerical predictions in terms of accelerations, displacements, dynamic earth pressures on the tunnel and dynamic internal forces of the tunnel lining are presented in the following sections compared to the experimental data, for the EQ4 earthquake scenario.

\subsection{Soil constitutive models}

- Visco-elastic analyses

For a first series of analyses, the soil non-linear behavior was modeled with a visco-elastic material, with properties (stiffness and damping) adequately selected, according to the shear deformation level, estimated for each earthquake scenario by means of 1D equivalent linear soil response analysis. The calculation was performed in the frequency domain using the code EERA [19]. The small-strain shear modulus $\left(\mathrm{G}_{\max }\right)$ was computed according to Hardin and 
Drenvich [20] that was found to give similar results, with the air-hammer tests (Figure 8a). The G- $\gamma$-D curves required for the analysis were estimated through a try and error procedure, checking different curves, so to achieve the best fitting of the numerical predictions with the experimental results (horizontal acceleration at the "free-field" array). The $\mathrm{G}_{\max }$ and the G- $\gamma$ $\mathrm{D}$, finally adopted, are presented in Figure 8, along with the maximum horizontal acceleration as computed with EERA and compared with the experimental data for EQ4 (Figure 8c).

In the 2D full dynamic analysis of the soil-tunnel system, the reduced soil shear modulus was introduced, following the distribution with depth, as computed with EERA. For this purpose, a user subroutine was encoded in ABAQUS. The damping was introduced in the Rayleigh type formulation as a mobilized value over all the soil depth. The mechanical properties of the soil as adopted for this case are summarized in Table 4.
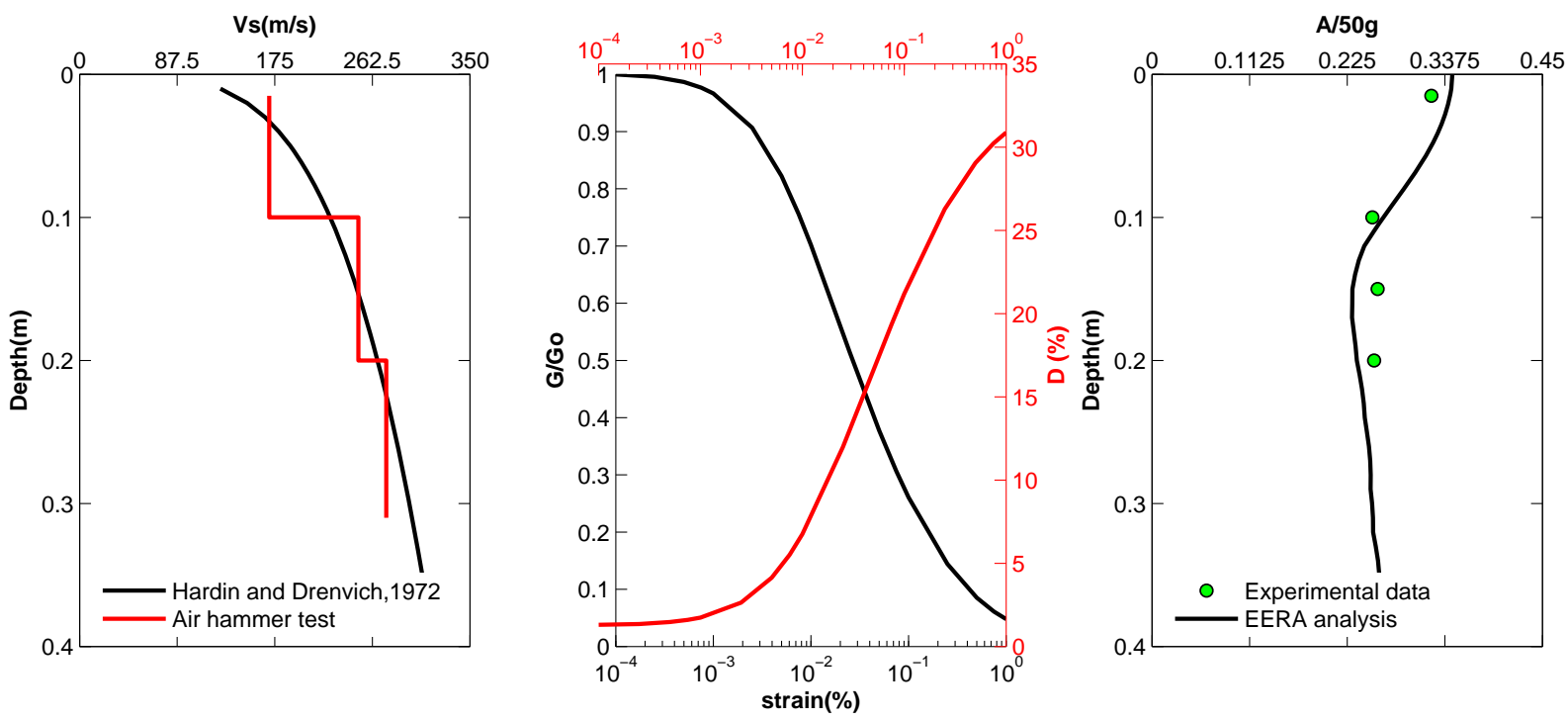

Figure 8: (a) $\mathrm{V}_{\text {so }}$ profile estimated from Hardin and Drenvich [20] compared to the air hammer test results, (b) G$\gamma$-D curves adopted in the analyses, (c) Comparison of the maximum horizontal acceleration along the "free field array" as estimated from the experimental data and computed from EERA analysis for EQ4

- Combined equivalent linear-elastoplastic approximation

To account of the soil plastic response (i.e. permanent deformations, residual values for the internal forces etc), a Mohr-Coulomb failure criterion was introduced in a second series of analyses. The elastic stiffness was kept reduced with respect to small-strain elastic stiffness, corresponding to the computed, from the $1 \mathrm{D}$ EQL response analysis, value. Regarding the strength parameters of the sand, the values were selected according to a literature reference for the specific fraction [21].

- Modified kinematic hardening model combined with a Von-Mises failure criterion and an associated plastic flow rule

For the final series of analyses, the soil non-linear behavior was modeled using a kinematic hardening model combined with a Von-Mises failure criterion and an associated plastic flow rule. The model is embedded in ABAQUS and modified through a user subroutine, as presented by Anastasopoulos et al. [22], to be applicable also for sands.

According to the model, the evolution of stress is defined as:

$$
\sigma=\sigma_{0}+\alpha
$$


where $\sigma_{0}$ corresponds to the stress at zero plastic strain, and $\alpha$, is the responsible for the kinematic evolution of the yield surface in the stress space, backstress. This is performed through a function $\mathrm{F}$ which defines the yield surface, as:

$$
F=f(\sigma-\alpha)-\sigma_{0}
$$

Accounting of the associated plastic flow rule, the plastic flow rule rate $\dot{\varepsilon}^{p l}$ is computed as:

$$
\dot{\varepsilon}^{p l}=\dot{\bar{\varepsilon}}^{p l} \frac{\partial F}{\partial \sigma}
$$

where $\dot{\bar{\varepsilon}}^{p l}$ is the equivalent plastic strain rate.

The evolution law comprises of an isotropic hardening component that describes the change of the equivalent stress defining the size of the yield surface $\sigma_{0}$ as a function of plastic deformation and a nonlinear kinematic hardening component, which describes the translation of the yield surface in the stress space. The latter is defined as an additive combination of a kinematic term and a relaxation term, which introduces the non-linearity. The evolution of the kinematic component of the yield stress is described according to the following formulation:

$$
\dot{a}=C \frac{1}{\sigma_{0}}(\sigma-\alpha) \dot{\bar{\varepsilon}}^{p l}-\gamma \alpha \dot{\bar{\varepsilon}}^{p l}
$$

where $\mathrm{C}$ is the initial kinematic hardening modulus (equal to the elastic stiffness) and $\gamma$ a parameter that describes the rate at which the kinematic hardening decreases with the increasing plastic deformation.

The evolution of the two hardening components (isotropic and kinematic) is illustrated in Figure 9 for unidirectional and multiaxial loading. The evolution law for the kinematic hardening component implies that the backstress $\alpha$ is contained within a cylinder of radius equal to $\sqrt{2 / 3} C / \gamma$. Since the yield surface remains bounded, any stress point must lie within a cylinder of a radius $\sqrt{2 / 3} \sigma_{y}$, where $\sigma_{y}$ is the maximum yield stress at saturation. To account of the confining pressure (i.e. for sands), $\sigma_{y}$ is defined as a function of the octrahedral stress and the friction angle $\varphi$ of the sand, as:

$$
\sigma_{y}=\sqrt{3}\left(\frac{\sigma_{1}+\sigma_{2}+\sigma_{3}}{3}\right) \sin \varphi
$$

where, $\sigma_{1}, \sigma_{2}, \sigma_{3}$ are the principles stresses. Since $\sigma_{y}=C / \gamma+\sigma_{0}, \gamma$ can be computed for cohesionless soils as:

$$
\gamma=\frac{C}{\sqrt{3}\left(\frac{\sigma_{1}+\sigma_{2}+\sigma_{3}}{3}\right) \sin \varphi-\sigma_{0}}
$$

The model parameters were calibrated against the small strain shear modulus distribution and the G- $\gamma$-D curves presented before. For this purpose, numerical simulations of cyclic simple shear tests were performed, for several shear strain levels, so to compute the shear modulus degradation and the increasing damping with the increasing strain. The results of these analyses are presented in Figure 9c, comparatively to the target G- $\gamma$-D curves. 
Table 4 summarizes the mechanical properties of the sand, finally adopted for all the constitutive models implemented.

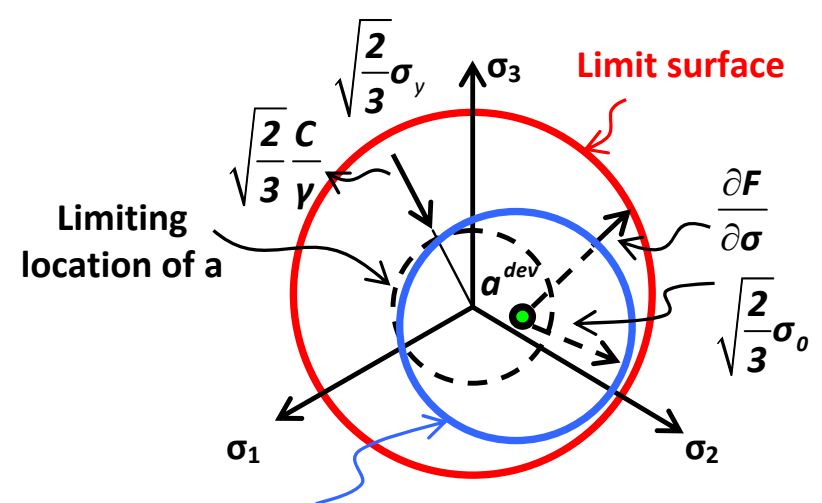

Yield surface

(a)

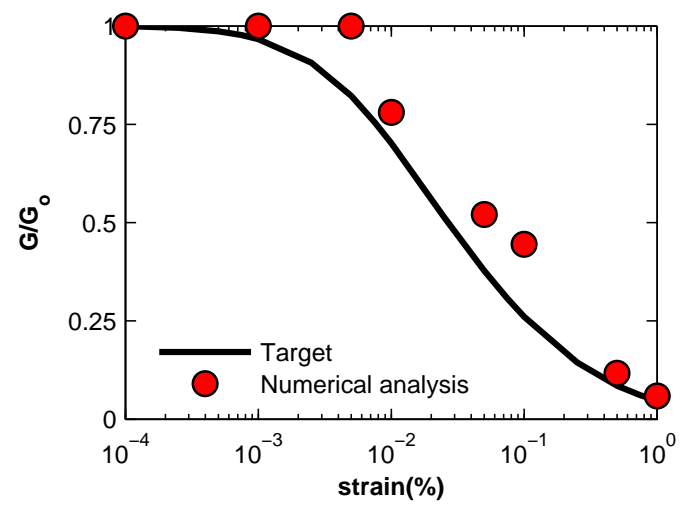

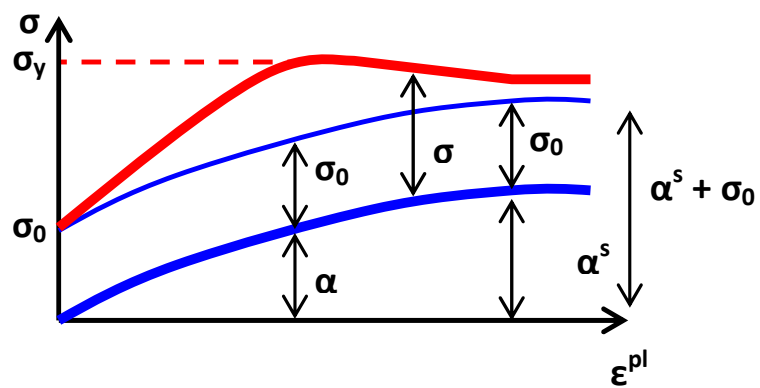

(b)

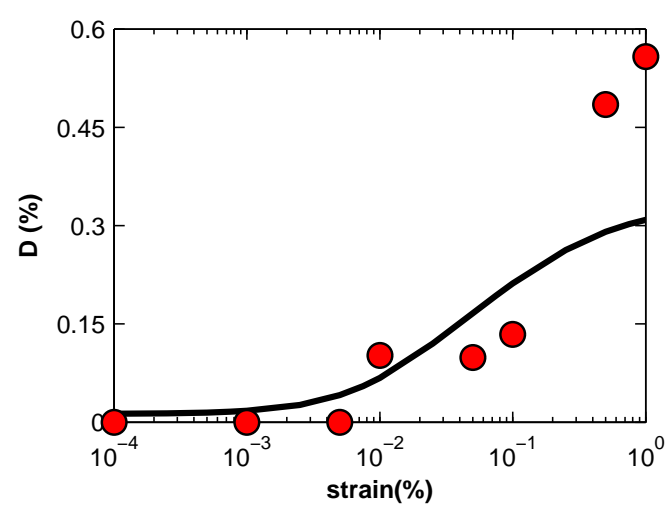

(c)

Figure 9: (a), (b) Nonlinear isotropic/kinematic hardening constitutive soil model: three-dimensional (a) and simplified one-dimensional (b) representation of the hardening law, (c) Calibration of the constitutive model against the $\mathrm{G} \gamma \mathrm{D}$ curves adopted for the specific test case

\begin{tabular}{llllllll}
\hline & $\begin{array}{l}\text { Shear modulus } \\
\mathrm{G}\end{array}$ & $\begin{array}{l}\rho \\
\left(\mathrm{t} / \mathrm{m}^{3}\right)\end{array}$ & $\mathrm{v}$ & $\begin{array}{l}\text { Damping } \\
(\%)\end{array}$ & $\begin{array}{l}\varphi \\
\left(^{\circ}\right)\end{array}$ & $\begin{array}{l}\Psi \\
\left({ }^{\circ}\right)\end{array}$ & $\begin{array}{l}\mathrm{c} \\
(\mathrm{kPa})\end{array}$ \\
\hline $\begin{array}{l}\text { Visco-elastic } \\
\text { analysis }\end{array}$ & Reduced distribution & 1.63 & 0.333 & $\begin{array}{l}15 \\
(\text { Rayleigh } \\
\text { type) }\end{array}$ & - & - & - \\
$\begin{array}{l}\text { Combined EQL } \\
\text { elastoplastic } \\
\text { analysis }\end{array}$ & Reduced distribution & 1.63 & 0.333 & $\begin{array}{l}15 \\
\text { (Rayleigh } \\
\text { type) } \\
10\end{array}$ & 40 & 12 & 2 \\
$\begin{array}{l}\text { Kinematic harden- } \\
\text { ing model }\end{array}$ & $\mathrm{G}_{\mathrm{o}}$ distribution & 1.63 & $0.333 \begin{array}{l}\text { (Rayleigh } \\
\text { type }+ \\
\text { hysteretic }\end{array}$ & 40 & - & 2 \\
\hline
\end{tabular}

Table 4: Soil mechanical properties adopted for each analysis case 


\section{EXPERIMENTAL DATA VS. NUMERICAL PREDICTIONS}

In this section we present, representative numerical results compared to the experimental data. The results are presented and discussed for EQ4 in terms of horizontal and vertical accelerations, dynamic earth pressures, dynamic internal forces of the model lining and soil surface settlements. They are shown at model scale, if not differently stated.

\subsection{Horizontal acceleration}

Figures 11-13 present windows of the computed and recorded acceleration time histories at several locations. The numerical predictions are in very good agreement with the records, with an exception for ACC14 (tunnel roof slab). The analysis where the kinematic hardening model is used to describe the non-linear behavior of the sand seems to slightly over predict the amplification of the horizontal acceleration with respect to the experimental data and the other analyses (Figure 10), but in general the representation of the actual behavior seems to be fair enough. The minor differences are mainly attributed to the difference between the assumed soil mechanical properties (stiffness and damping) and their actual values during the test.

The large difference between the recorded and the computed horizontal acceleration on the roof slab (ACC14) is probably attributed to a recording error, as the analyses are reproducing quite efficiently the acceleration both at the soil surface above the tunnel and on the other locations of the tunnel (middle of the right wall, invert slab).

\subsection{Fundamental frequency of the soil deposit}

Figure 14 presents the transfer functions (at prototype scale) as computed along the "freefield" vertical array of the accelerometers, by the experimental data and the numerical analyses. Both experimental and the numerical results are reasonable well compared indicating a fundamental frequency of about $2 \mathrm{~Hz}$ for the soil deposit.
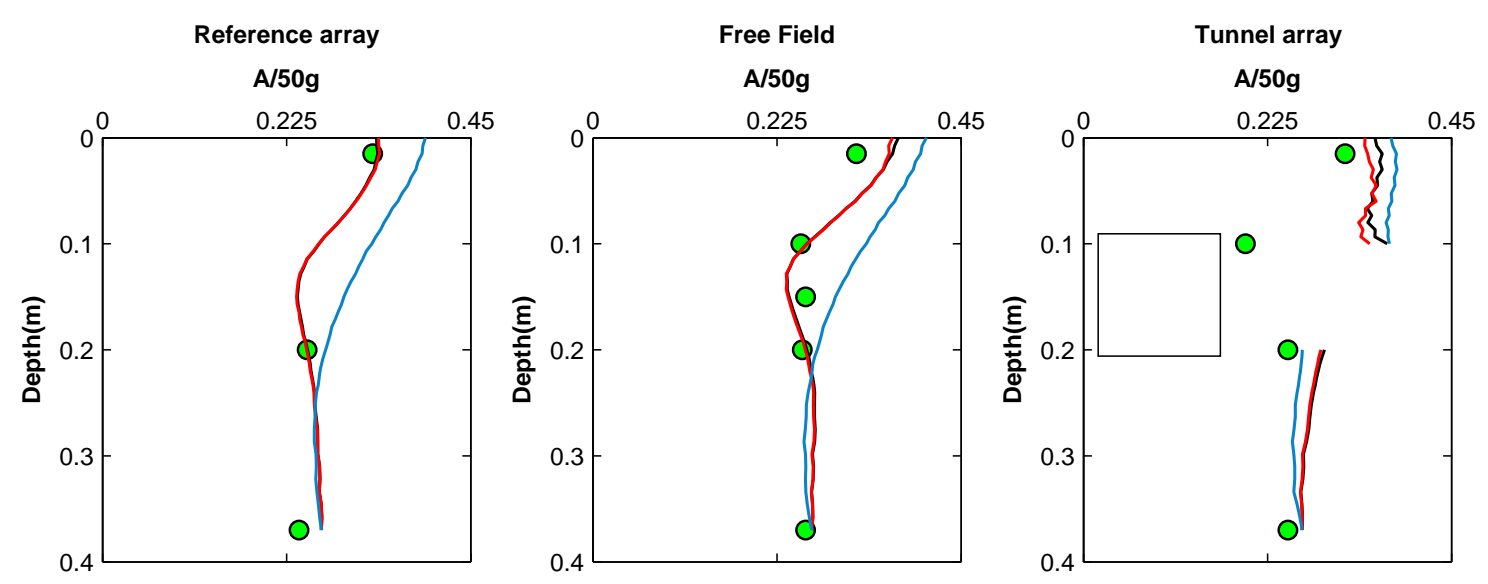

Figure 10: Maximum horizontal acceleration along vertical accelerometers arrays; Experimental data vs. numerical predictions (green circle points: experimental data, black solid line: visco-elastic analysis, red solid line: combined EQL elastoplastic analysis, blue solid-line: kinematic hardening model) 

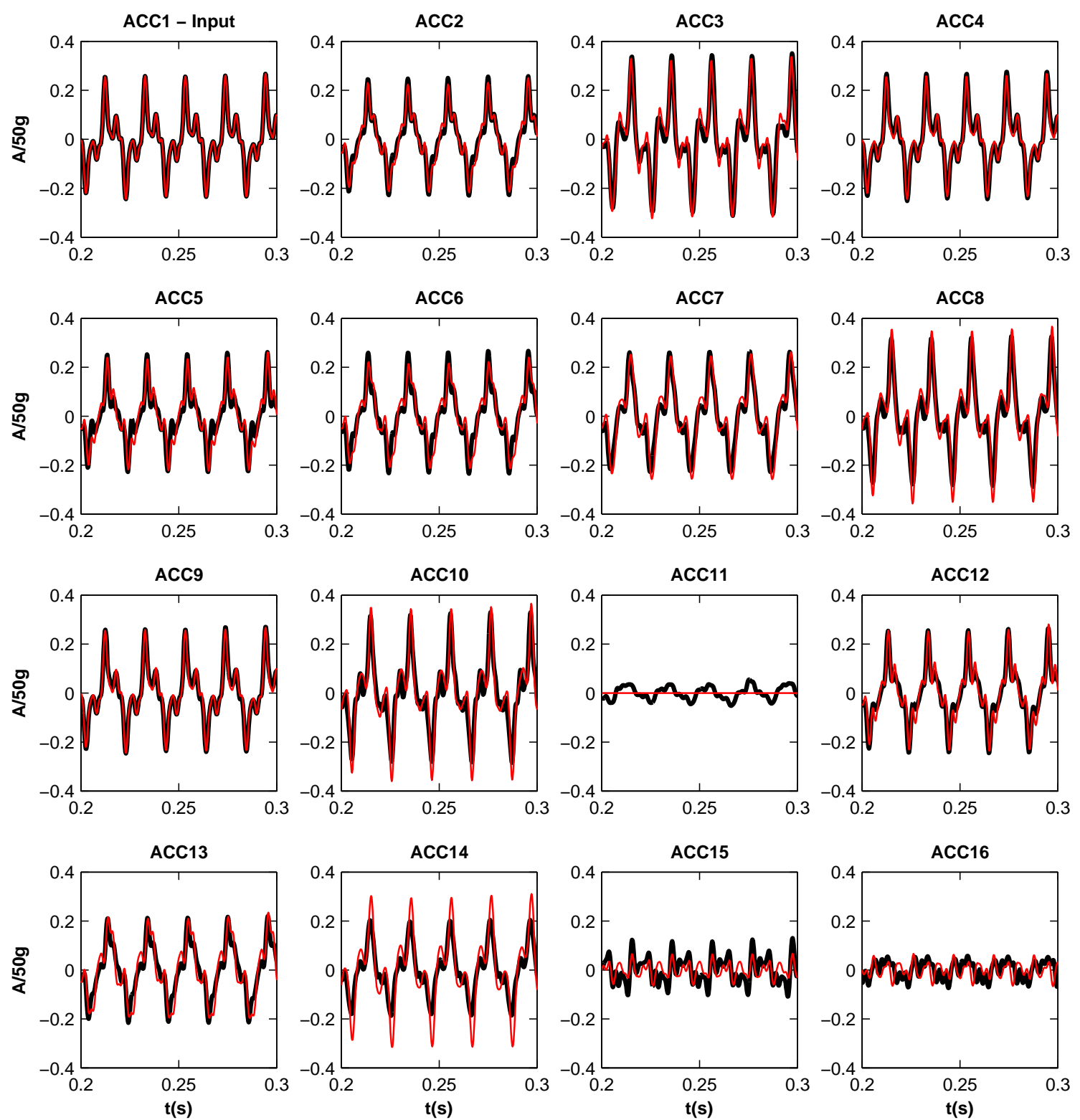

Experimental data

- Numerical prediction

Figure 11: Windows of acceleration time histories for EQ4; Experimental records vs. visco-elastic analysis results 

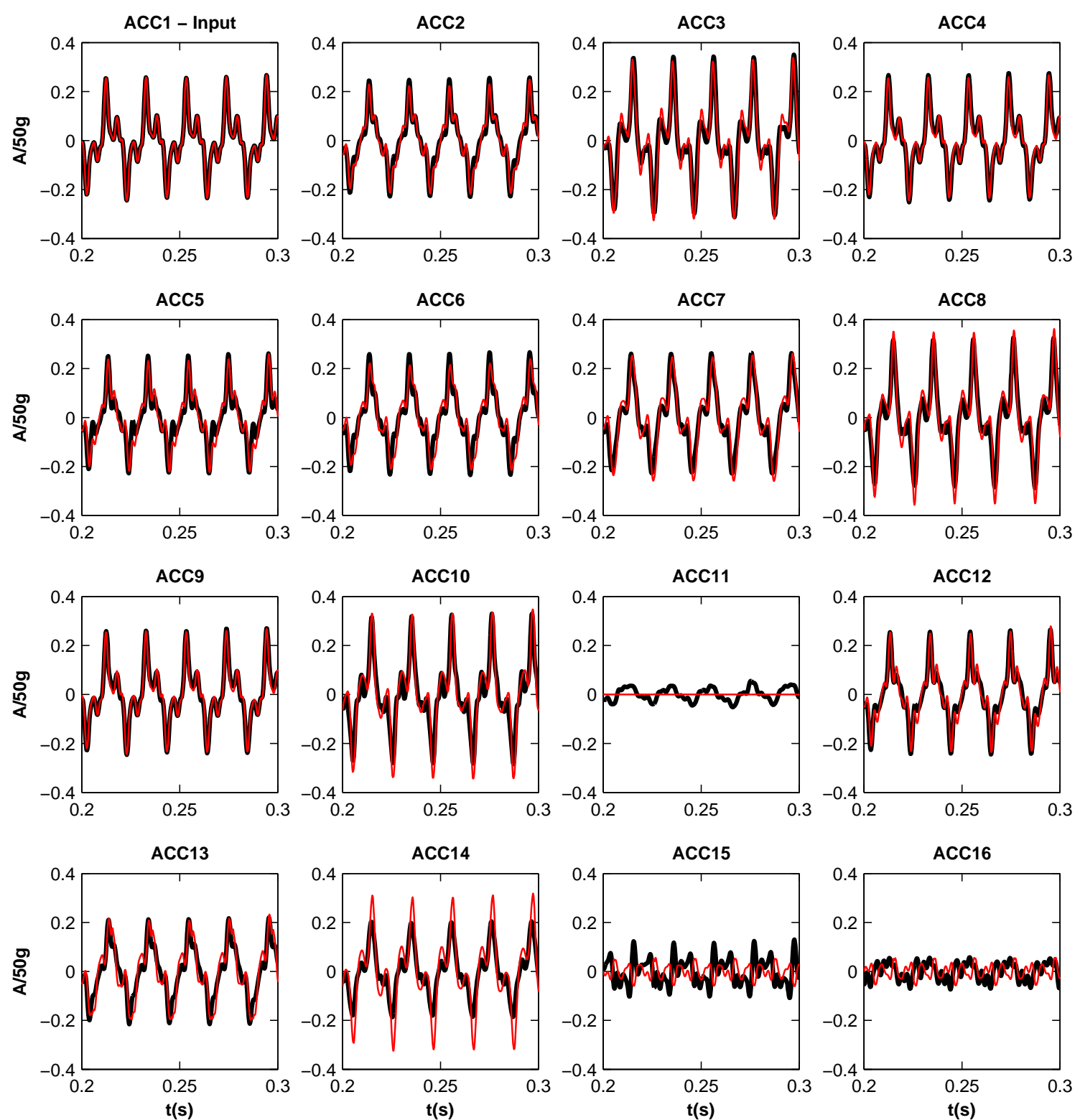

Experimental data

Numerical prediction

Figure 12: Windows of acceleration time histories for EQ4; Experimental records vs. combined EQLelastoplastic analysis results 

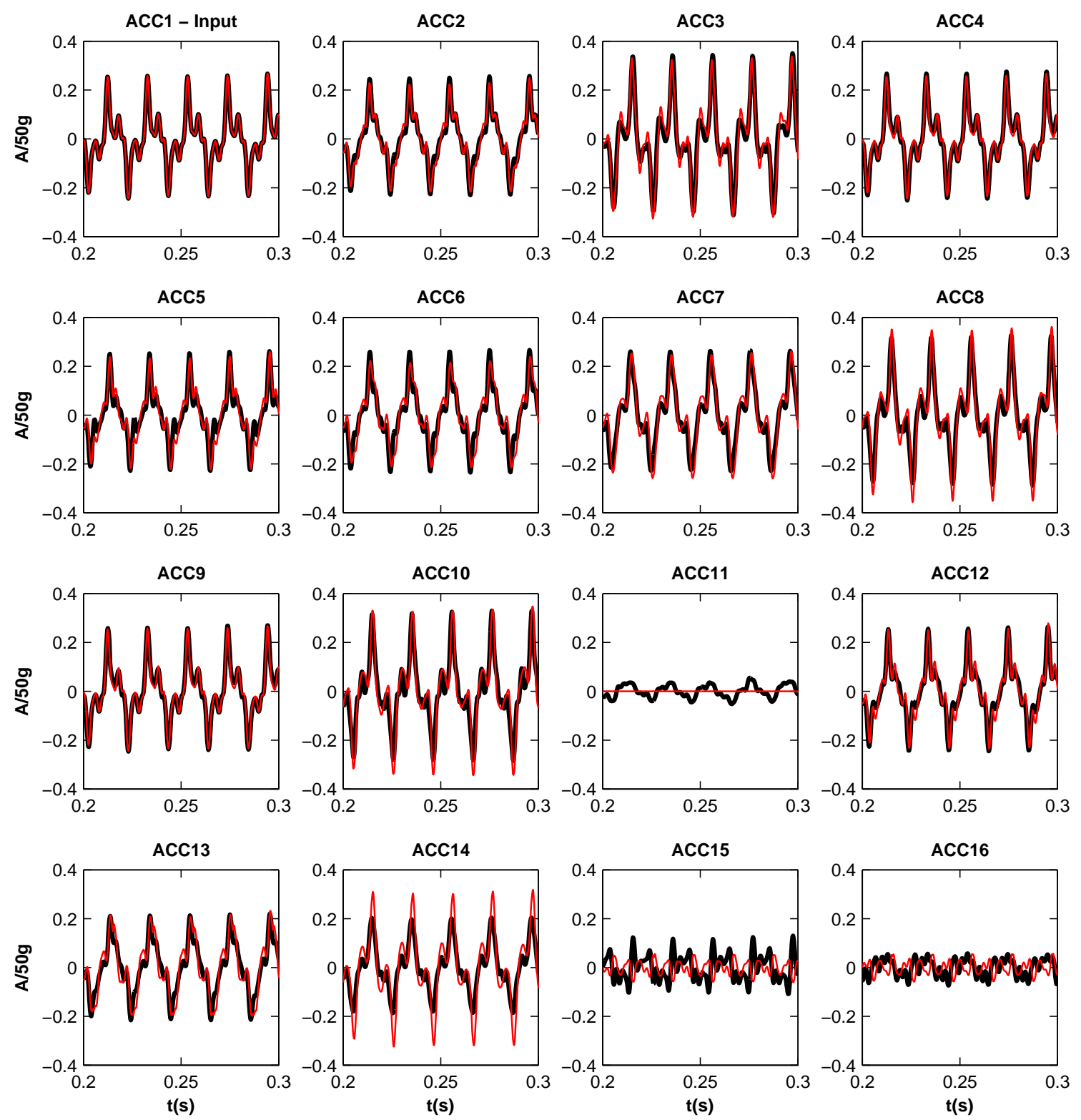

Experimental data

- Numerical prediction

Figure 13: Windows of acceleration time histories for EQ4; Experimental records vs. kinematic hardening model results 


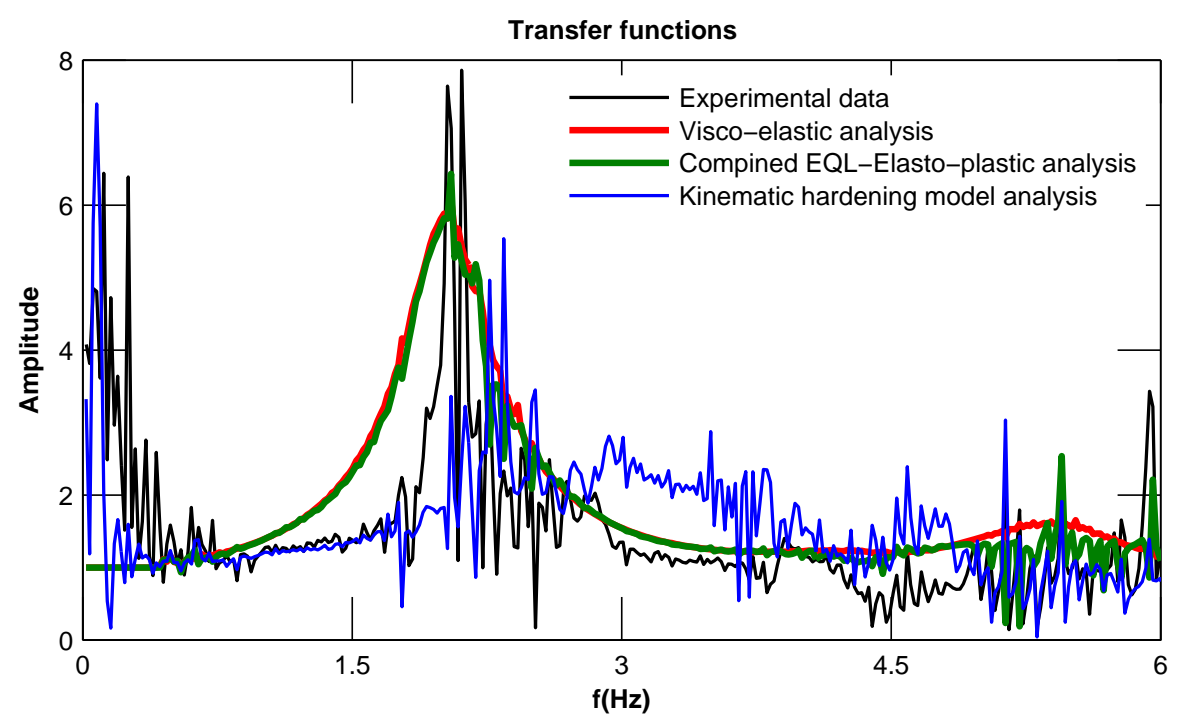

Figure 14: Transfer functions along the "free field" vertical array of accelerometers for EQ4

\subsection{Vertical acceleration}

Small values of vertical acceleration were recorded near the soil deposit base. This is probably attributed to the reduced parasitic yawing movement of the ESB box on the shaking table, which is not modeled in the numerical analyses. Noticeable vertical accelerations were recorded and also computed at the sides of the tunnel roof slab (Figure 15). In both recorded and computed values we observe an out of phase response indicating a rocking mode of vibration for the tunnel, except the prevailing racking distortion. The reproduction of this mode of vibration by the numerical analysis, even without the simulation of the yawing movement of the ESB box, indicates that the yawing movement probably amplifies this behavior but in any case it is present. It is noted that this mode of vibration is usually precluded from the simplified analysis methods.
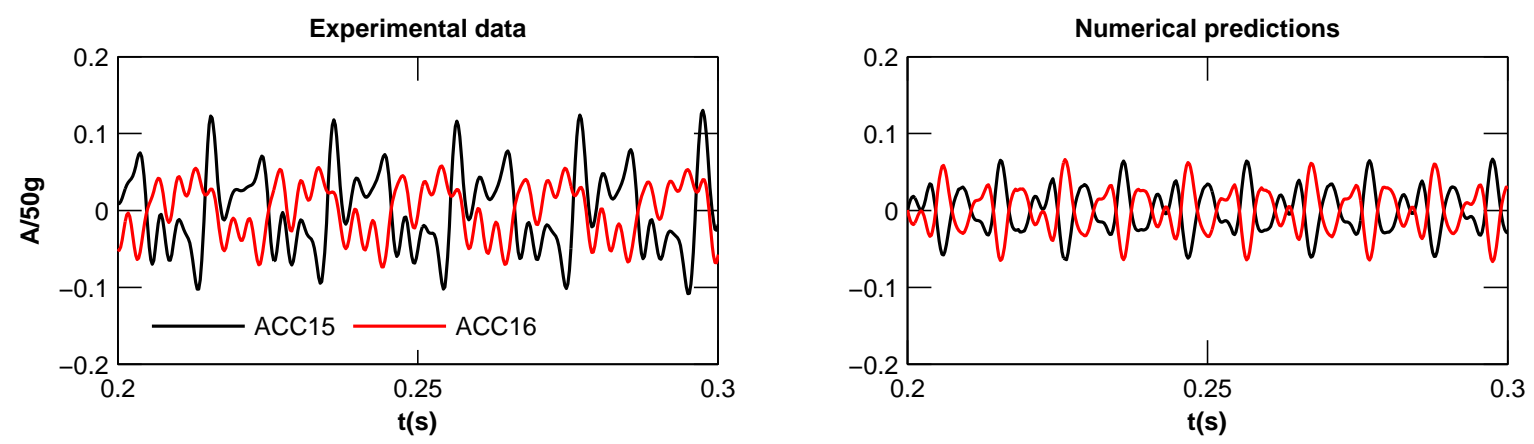

Figure 15: Windows of vertical acceleration time histories for EQ4; (a) Experimental records. (b) Visco-elastic analysis results

\subsection{Soil surface settlements}

Figure 16 presents the recorded and computed soil surface settlements (in model scale) during EQ4 test. The numerical analyses generally underestimate the soil settlements, which are anyway quite small (less than $0.6 \mathrm{~mm}$ for this case), due to the high relative density of the soil deposit. However it should be noticed that the experimental results maybe biased to some 
extend by the way the LVDTs are fixed to the gantries of the box and by the small bending of these gantries caused by the strong gravity forces.
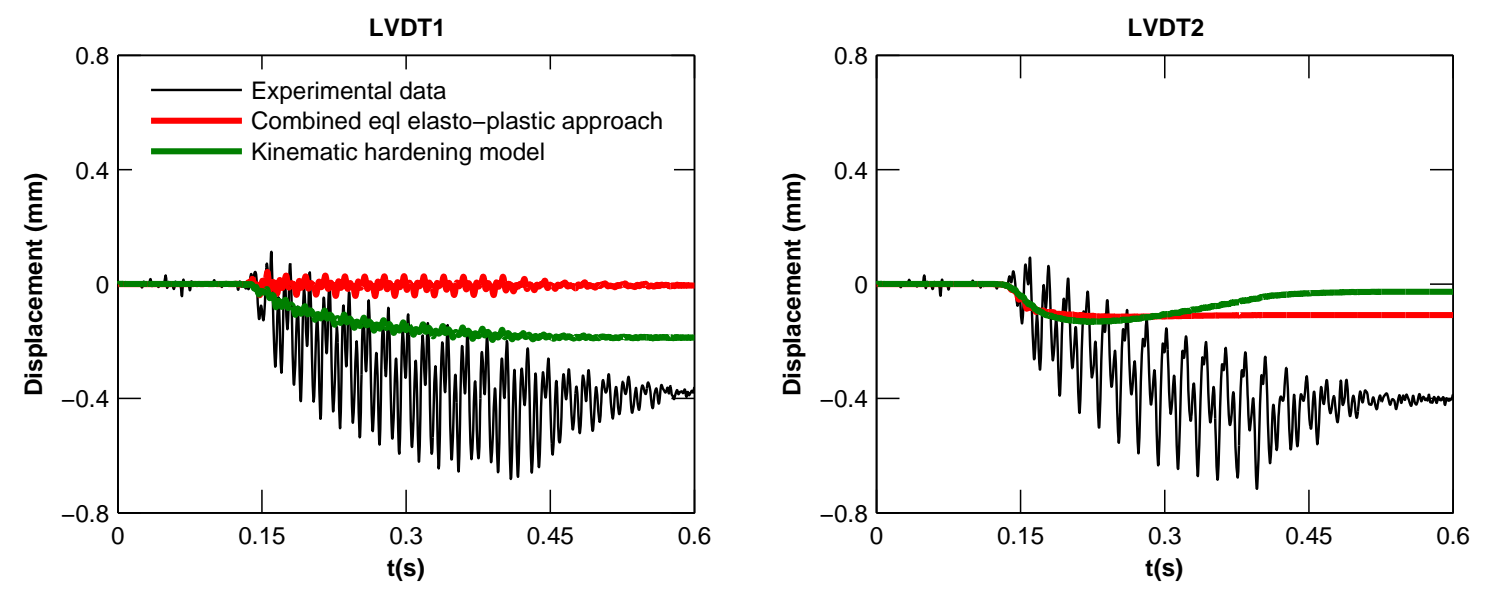

Figure 16: Soil surface settlements, EQ4; Experimental records vs. numerical predictions

\subsection{Dynamic earth pressures}

The computed and recorded dynamic earth pressures close to the invert slab-left wall corner and at the middle of the left side wall are presented in Figure 17.
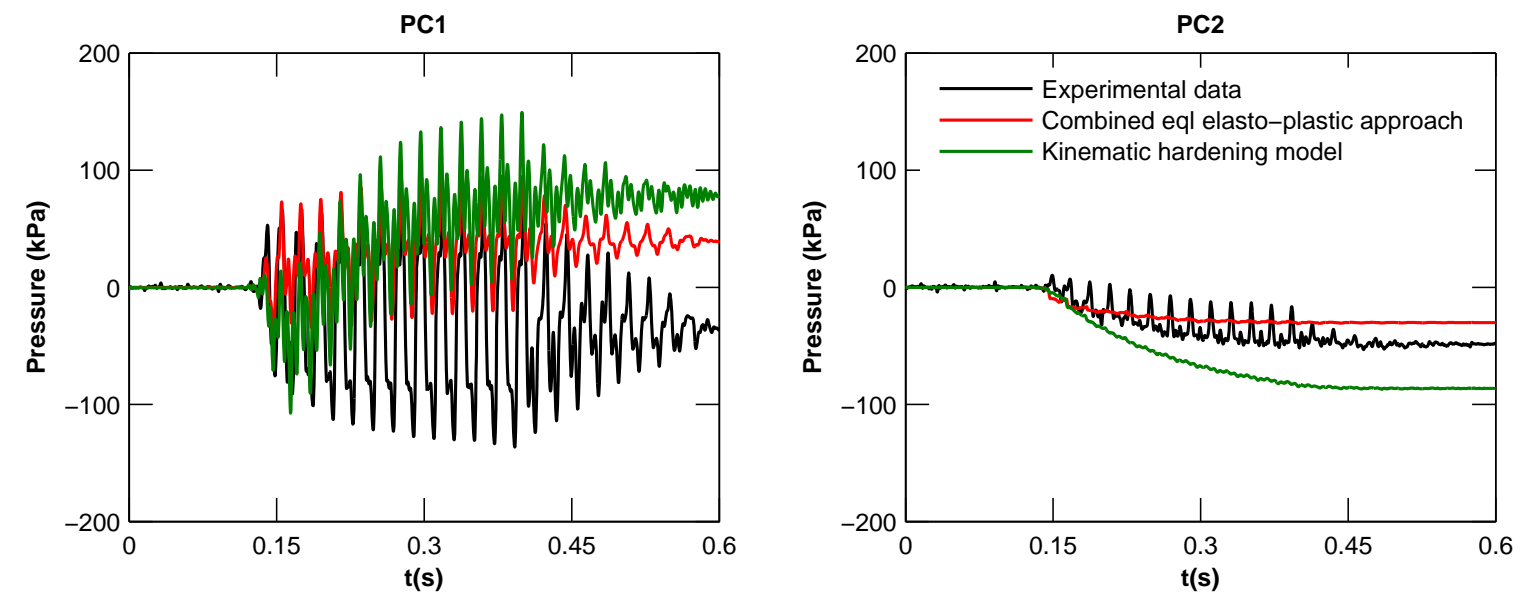

Figure 17: Dynamic earth pressures, EQ4; Experimental records vs. numerical predictions

Looking at the experimental data, three phases may be indentified, namely a transient stage, a steady state stage and finally a post-earthquake residual stage. During the first few cycles of loading, seismic earth pressures are building up. Then in the steady state stage the earth pressures are oscillating around a mean value. During this step, the pressures are shifting from active to passive state due to the tunnel oscillation. Finally, in the post-earthquake stage residual stresses are recorded on the tunnel lining. This behavior has been also reported in other similar centrifuge tests [23]. The dynamic increments of the pressures were found to be larger near the corner, while at the middle of the side wall the increments were much smaller due to the 
flexibility of the wall at this location. Generally, this complex behavior is attributed to the soil plastic deformations and the soil densification during shaking.

Similar observations were drawn from the numerical analyses, as the three aforementioned stages were reproduced to some extend. The differences in terms of dynamic increments (i.e. semi-amplitude of cycles in the time histories) and residual values are mainly attributed to differences between the adopted soil and soil-tunnel interface mechanical properties (i.e. soil stiffness and strength near the tunnel, interface coefficient of friction) and the actual values. It is noted that these parameters are highly interrelated. For example, a more "rigid" connection of the tunnel with the soil (i.e. larger coefficient of friction) can affect the soil stiffness degradation near the tunnel resulting in different stress redistribution in the soil around the tunnel. To this end, proper estimation of these parameters is of major importance.

\subsection{Dynamic internal forces}

The recorded and computed dynamic internal forces are presented in Figures 18-19.

Regarding the bending moments records, similar behavior to the earth pressures response is observed, as the three aforementioned stages are also reported in this case. This cumulative response has also been observed during dynamic centrifuge tests performed on circular tunnel models embedded in dry sand [24]. Similar response is observed in the numerical analyses. More specifically, both the combined EQL elasto-plastic analysis and the kinematic hardening model, seem to reproduce the three stages mentioned before (transient stage, steady state stage and post-earthquake residual stage), with the latter predicting much higher residual values after shaking.
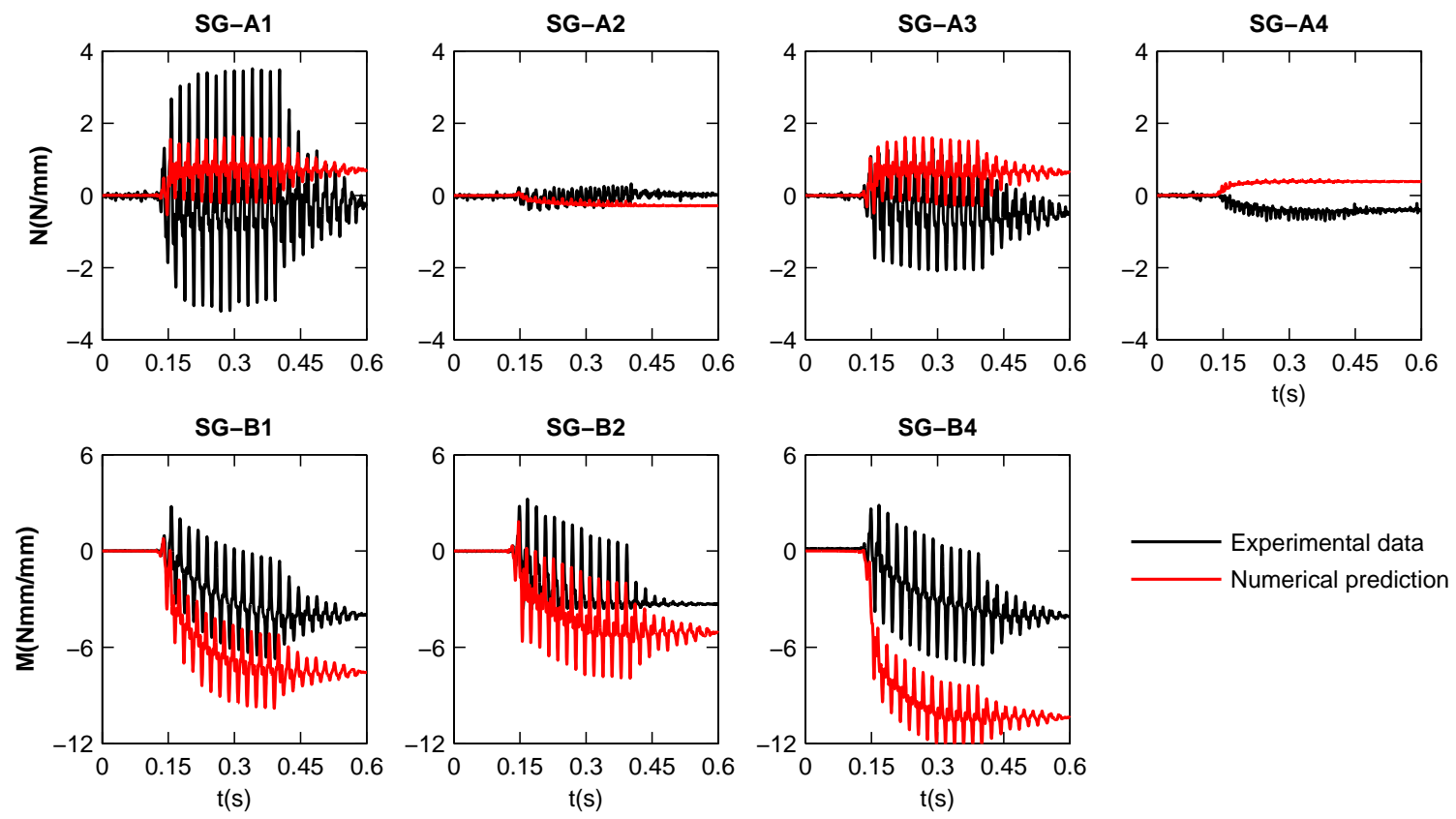

Figure 18: Dynamic internal forces, EQ4; Experimental records vs numerical predictions for the combined EQL elasto-plastic model 
Residual values were also observed for the axial forces. These residuals, that were generally smaller than the bending moment ones, found to be larger at the slabs. They can be attributed to the small soil densification as well as to the possible sliding effects on the soil-tunnel interface and to some extent to the soil non-linear behavior.

It is also important to notice that the dynamic axial forces recorded and computed on the sidewalls of the model, were out of phase (Figure 20), indicating again the rocking mode of vibration for the tunnel, as also mentioned for the vertical accelerations.
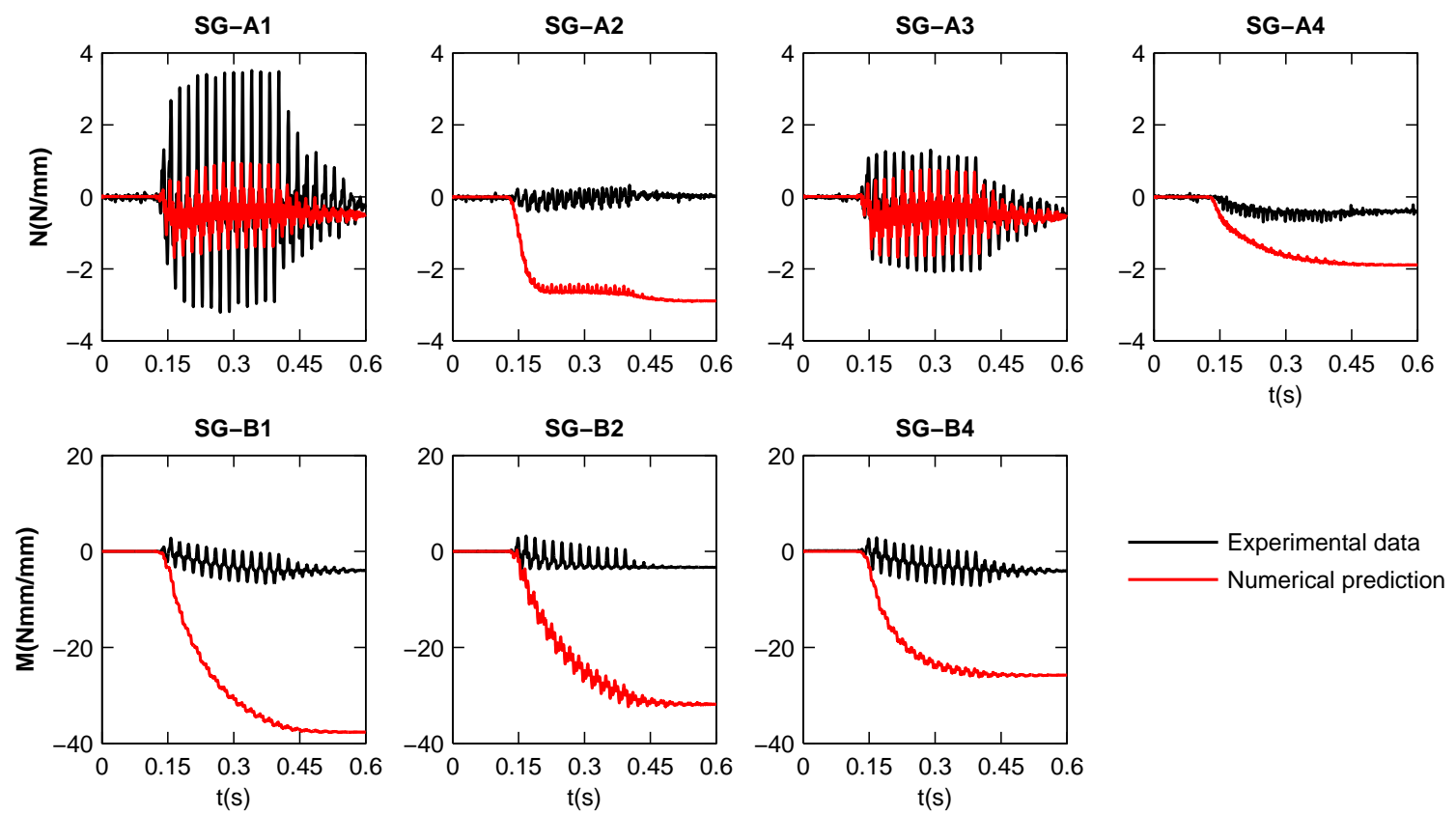

Figure 19: Dynamic internal forces, EQ4; Experimental records vs. numerical predictions for the kinematic hardening model
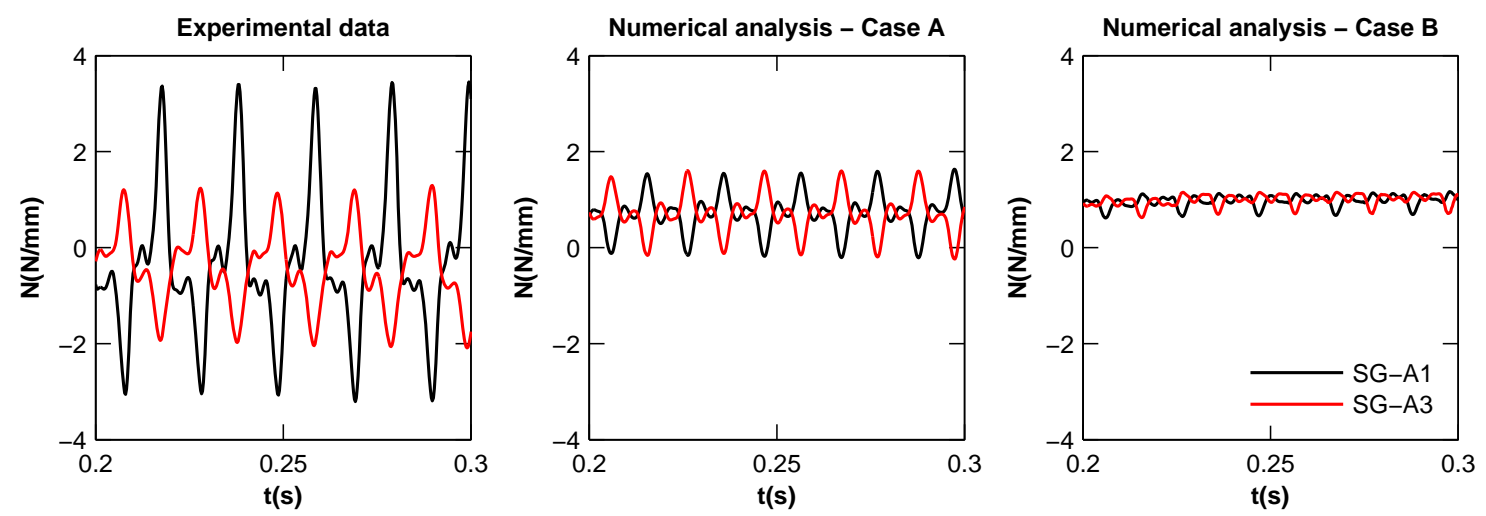

Figure 20: Time windows of the recorded and computed dynamic axial forces of the side walls; Case A: Combined EQL elasto-plastic analysis, Case B: Combined EQL elasto-plastic analysis - full slip conditions

Similar to the earth pressures, the differences between the recorded response and the numerical predictions are attributed to the differences between the adopted and the actual values of the soil and soil-tunnel interface mechanical properties and to some extend to their interre- 
lation. To check this hypothesis, we run the combined EQL elastoplastic analysis assuming full slip conditions for the soil-tunnel interface (no shear stress is transferred from soil to the tunnel). Figure 21 compares the recorded internal forces with the numerical predictions for this case. It can be observed that the dynamic increments of the axial forces are highly reduced with respect to the previous case (finite slip conditions), while the computed bending moments come closer the experimental data. The interrelation of the soil non-linear behavior and the soil-tunnel interface characteristics can also be observed in Figure 22, where the soil plastic strains as computed by the non-linear analyses are presented on the deformed shaped models. Actually, we can see differences on the soil plastic deformations distributions around the tunnel for the different assumptions regarding the soil-tunnel interface. For the full-slip conditions the soil plastic deformations are smaller near the walls and larger above the tunnel, with respect to the finite slip case.

\section{CONCLUSIONS}

The paper presented some representative results from a test of a series of dynamic centrifuge tests that were conducted on a square tunnel model embedded in dry sand. The case was also numerically modeled using different models for the soil behavior and soil-tunnel interface properties. Numerical predictions were compared to the experimental data. The main conclusions drawn may be summarized in the following:
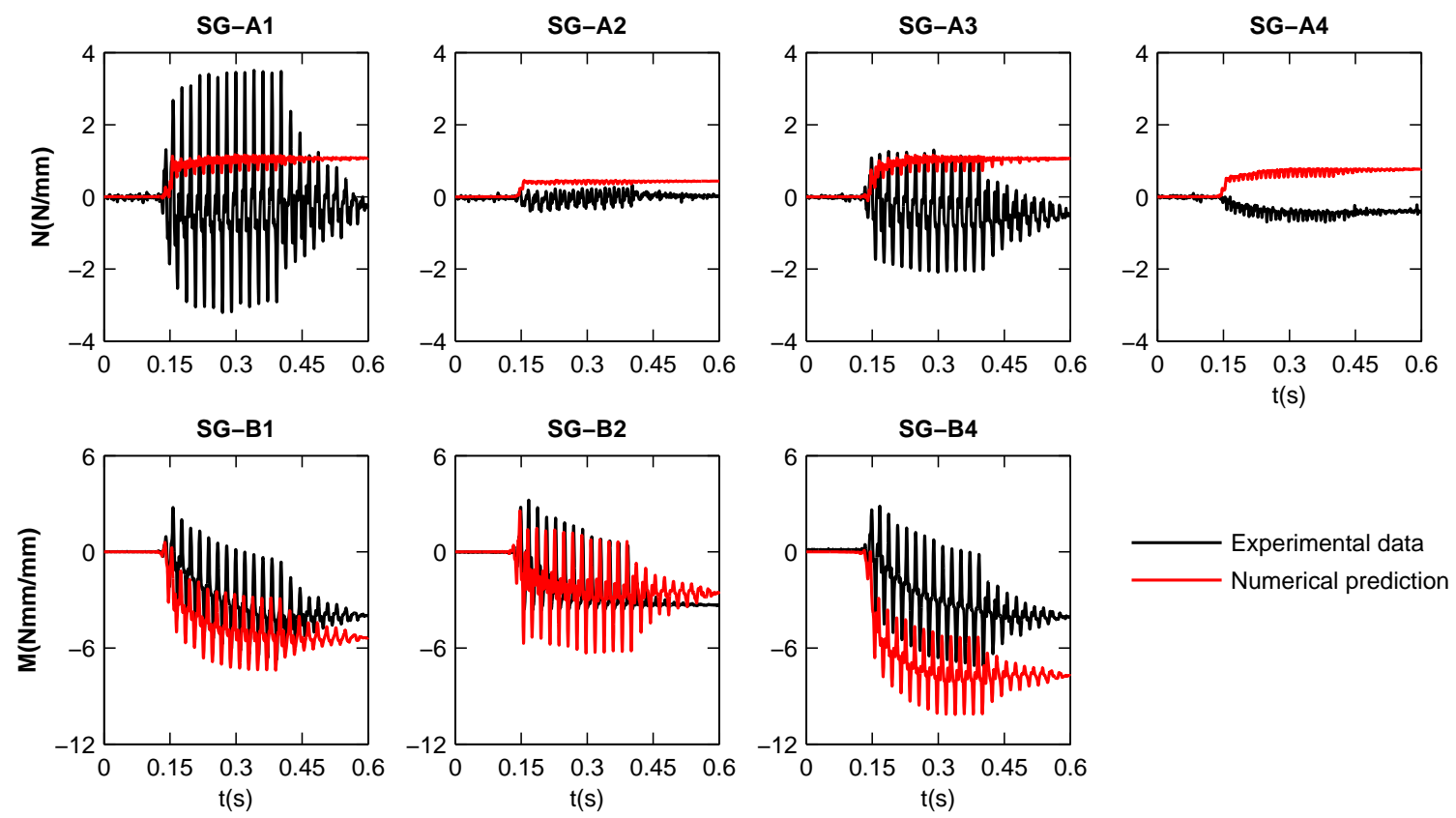

Figure 21: Dynamic internal forces, EQ4; Experimental records vs. numerical predictions for the combined eql elasto-plastic model assuming full slip conditions 


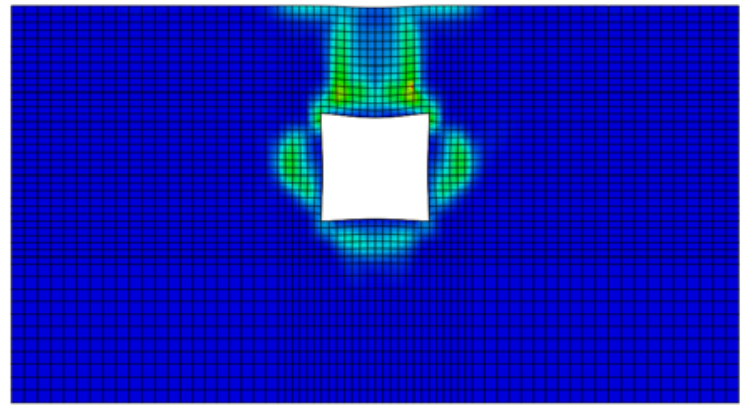

(a)

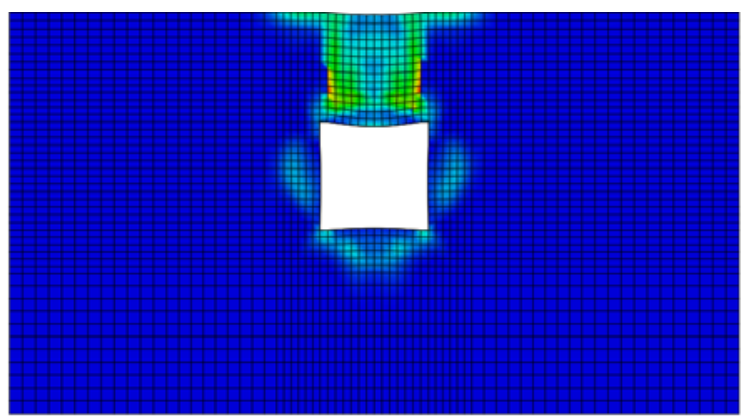

(c)

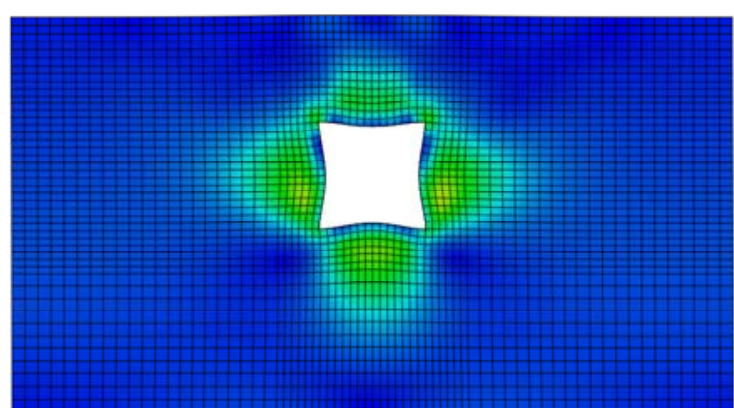

(b)

PE, Max. In-Plane Principal (Avg: 75\%)

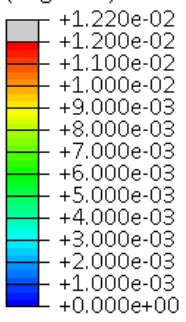

Figure 22: Soil plastic strains around the tunnel: (a) Combined EQL elasto-plastic analysis, (b) Kinematic hardening model, (c) Combined EQL elasto-plastic analysis - full slip conditions

- The horizontal accelerations recorded at several locations, are amplified towards the surface. This amplification was efficiently reproduced by the numerical analyses.

- Vertical acceleration-time histories recorded on the sides of the model's roof slab were out of phase indicating a rocking mode of vibration for the tunnel. This response was verified by the numerical analysis.

- The recorded earth pressure increments are higher at the slab-wall corner due to the larger rigidity of the model at this location compared to the middle of the wall. Residual values were observed after each shake that can be attributed to the soil plastic deformations and to a small amount of soil densification during shaking that can cause stress redistribution. This general trend was also numerically reproduced.

- The dynamic bending moments follow the same trend as for the dynamic earth pressures. Large residual values were detected after each shake, as a result of cumulative strains during the shaking.

- Smaller residuals were observed for the dynamic axial forces after shaking. In addition to the aforementioned parameters that can cause these residual values, a small amount of sliding on the soil-tunnel interface is also affecting this behavior.

- The numerical models used herein, generally reproduced the general trends observed from the recorded dynamic internal forces. The differences between the computed and the recorded values are attributed to the differences between the adopted soil and soiltunnel interface mechanical properties compared to the actual values and to some extend to their interrelations. Errors related with the records (i.e. calibration of the instruments) can also cause differences to some extend.

- Generally, we concluded that numerical models may reproduce accurately, considering all kind of uncertainties involved, the recorded response of a rectangular tunnel in the 
centrifuge. Further analysis of the recorded data and better evaluation of the soil and structure properties during shaking are expected to provide even better insight of the complex behavior of this kind of structures.

\section{ACKNOWLEDGMENTS}

The research leading to these results has received funding from the European Community's Seventh Framework Programme [FP7/2007-2013] for access to the University of Cambridge under grant agreement $\mathrm{n}^{\circ} 227887$ (SERIES Research Program). The technical support received by the Technicians of the Schofield Centre is gratefully acknowledged. The authors would like to acknowledge Assistant Professor Ioannis Anastasopoulos and Dr. Fani Gelagoti, for the fruitful discussions they had with for the implementation of the kinematic hardening model.

\section{REFERENCES}

[1] S. Sharma, W.R. Judd, Underground opening damage from earthquakes. Eng Geology 30, 263-276, 1991.

[2] M. Power, D. Rosidi, J. Kaneshiro, S. Gilstrap, S.J. Chiou, Summary and evaluation of procedures for the seismic design of tunnels. Final Report for Task 112-d-5.3(c). National Center for Earthquake Engineering Research, Buffalo, New York, 1998.

[3] H. Iida, T. Hiroto, N. Yoshida, M. Iwafuji, Damage to Daikai subway station. Special issue on geotechnical aspects of the January 171995 Hyogoken-Nanbu earthquake. Soils Found, 283-300, 1996.

[4] Y.M.A. Hashash, J.J. Hook, B. Schmidt, J.I.-C. Yao, Seismic design and analysis of underground structures. Tunneling Undergr Space Technol, 16 (2), 247-293, 2001.

[5] Special Issue of Soil and Foundations. Selected photographs on the 1995 HyogokenNambu earthquake. Special issue on geotechnical aspects of the January 171995 Hyogoken-Nambu earthquake. Japanese Geotechnical Society, 1996.

[6] J.N. Wang, Seismic Design of Tunnels. Parson Brinckerhoff, New York, 1993.

[7] J. Penzien, Seismically induced racking of tunnel linings. Earthquake Eng Structural Dyn, 29, 683-691, 2000.

[8] ISO 23469, Bases for design of structures - Seismic actions for designing geotechnical works. ISO International Standard. ISO TC 98 / SC3 /WG10, 2005.

[9] F.W.H.A., Technical manual for design and construction of road tunnels - Civil elements. U.S. Department of transportation. Federal Highway Administration. Publication No. FHWA-NHI-09-010, 2009.

[10] K. Pitilakis K, G. Tsinidis, Performance and seismic design of underground structures. II International Conference on Performance Based Design in Earthquake Geotechnical Engineering (State of the art lecture), Taormina, Italy, May, 2012.

[11] S.P.G. Madabhushi, A.N. Schofield, S. Lesley, A new stored angular momentum (SAM) based actuator. T. Kimura, O. Kusakabe, J. Takemura, eds. Centrifuge 98, Tokyo, Japan. A.A. Balkema publishers 111-116, 1998. 
[12] A.N. Schofield, X. Zeng, Design and performance of an equivalent shear beam (ESB) container for earthquake centrifuge modelling. Technical Report, CUED/DSoils/I'R245, Cambridge University, 1992.

[13] H. Mitrani, Liquefaction Remediation Techniques for Existing Buildings. PhD Thesis, University of Cambridge, 2006.

[14] Y. Zhao, K. Gafar, M.Z.E.B Elshafie, A.D Deeks, J.A. Knappett, S.P.G. Madabhushi, Calibration and use of a new automatic sand pourer. Zhang, Wang eds. Physical Modelling in Geotechnics - 6th ICPMG '06, Taylor \& Francis Group, London, 2006.

[15] B. Ghosh, S.P.G. Madabhushi, An efficient tool for measuring shear wave velocity in the centrifuge. R. Phillips, P.J. Guo, R. Popescu eds. Int Conf on Physical Modelling in Geotechnics, St Johns, NF, Canada. AA Balkema publishers, 119-124, 2002.

[16] G. Tsinidis, C. Heron, S.P.G. Madabhushi, K. Pitilakis, M. Stringer, SERIES TA Project: TUNNELSEIS: Investigation of several aspects affecting the seismic behaviour of shallow rectangular underground structures in soft soils, Technical Report (Under development), 2013.

[17] A.N. Schofield, Cambridge Geotechnical Centrifuge Operations. Geotechnique 30, (3), 227-268, 1981.

[18] ABAQUS Analysis User's Manual - Volumes I - IV, Dassault Systèmes, SIMULIA Inc, USA \{Computer code\}

[19] J.P. Bardet, K. Ichii, C.H. Lin, EERA: A computer program for equivalent-linear earthquake site response analyses of layered soil deposits. University of California, Department of Civil Engineering, 2000.

[20] B.O. Hardin, V.P. Drnevich, Shear modulus and damping in soils: design equations and curves. J Soil Mech Found Division 98 (SM7), 667-692, 1972.

[21] T. Schanz, P.A. Vermeer, Angles of friction and dilatancy of Sand. Geotechnique, 46 (1), 145-151, 1996.

[22] I. Anastasopoulos, F. Gelagoti, R. Kourkoulis, G. Gazetas, Simplified constitutive model for simulation of cyclic response of shallow foundations: Validation against laboratory tests. J Geotech Geoenvironmental Eng, 137 (12), 1154-1168, 2011.

[23] U. Cilingir, S.P.G. Madabhushi, A Model Study on the Effects of Input Motion on the Seismic Behavior of Tunnels. J. Soil Dyn Earthquake Eng, 31, 452-462, 2011.

[24] G. Lanzano, E. Bilotta, G. Russo, F. Silvestri, S.P.G. Madabhushi, Centrifuge modeling of seismic loading on tunnels in sand. Geotech Testing J, 35, 2012. 